\title{
Pulmonary manifestations of immunoglobulin G4-related sclerosing disease
}

\author{
J.H. Ryu*, H. Sekiguchi* and E.S. Yi ${ }^{\#}$
}

ABSTRACT: Immunoglobulin (Ig)G4-related sclerosing disease (ISD) (also called IgG4-related systemic disease, IgG4-related disease or hyper-IgG4 disease) is a recently described systemic fibroinflammatory disease associated with elevated circulating levels of IgG4.

Although initial descriptions of this disorder focused on its pancreatic presentation (autoimmune pancreatitis), it has become apparent that ISD is a systemic disease with many facets. The lesion of ISD is characterised by lymphoplasmacytic inflammation, fibrosis, phlebitis and increased numbers of IgG4-positive plasma cells. The disease can either be localised to one or two organs, or be present with diffuse multi-organ disease. Furthermore, lesions in different organs can present simultaneously or metachronously. In the thorax, lesions associated with ISD have been described in the lung parenchyma, airways and pleura, as well as the mediastinum.

Data published to date suggest that ISD may account for a portion of various fibroinflammatory conditions of unknown cause encountered in the chest, including inflammatory pseudotumours, idiopathic interstitial pneumonias, fibrosing mediastinitis, inflammatory pleural lesions and, occasionally, airway disease.

Some aspects of pulmonary manifestations attributed to ISD remain controversial and additional studies are needed to clarify the relationship along with the increasing relevance of this disorder to pulmonary medicine.

KEYWORDS: Immunoglobulin G4, interstitial lung disease, lymphadenopathy, pulmonary inflammation

I mmunoglobulin (Ig)G4-related sclerosing disease (ISD) is a recently described systemic fibroinflammatory disease associated with elevated circulating levels of IgG4. This disorder has also been referred to as IgG4-related systemic disease, IgG4-related disease and hyper-IgG4disease [1-4]. Although consensus has not been achieved on the name of this disorder, we and others have preferred the term ISD based on the characteristic fibrosclerosing tendency of the lesions associated with this disease. Initial reports relating to ISD focused on its pancreatic manifestations, for which the term autoimmune pancreatitis was initially proposed in 1995 by YOSHIDA et al. [5]. In 2001, HAMANO et al. [6] reported high serum IgG4 concentrations in 20 patients with autoimmune pancreatitis (or sclerosing pancreatitis). It is now realised that a substantial portion (40-60\%) of autoimmune pancreatitis cases represent the pancreatic manifestation of an ISD, i.e. IgG4-related sclerosing disease [7]. Studies published over the past few years have documented the association of a high serum IgG4 level with the characteristic lesion of dense lymphoplasmacytic infiltrates containing IgG4-positive plasma cells in various organs, including the bile duct (sclerosing cholangitis) [8-11], salivary gland (sclerosing sialadenitis) [2, 9-11], lacrimal gland (sclerosing dacryoadenitis) $[2,10,11]$, liver (IgG4-hepatopathy) [2], kidney (inflammatory pseudotumour) $[2,10]$, retroperitoneum (retroperitoneal fibrosis) [2, 9-11], aorta (inflammatory aneurysm) [2, 12], and lymph nodes $[2,9,13]$, as well as the lung $[2,10,14-17]$. ISD can be localised to one or two organs or be present with diffuse systemic disease [1-3, 18-20]. Furthermore, lesions in different organs can present simultaneously or metachronously.
AFFILIATIONS

*Division of Pulmonary and Critical Care Medicine, and

\#Division of Anatomic Pathology,

Mayo Clinic, Rochester, MN, USA.

CORRESPONDENCE

J.H. Ryu

Division of Pulmonary and Critical

Care Medicine

Gonda 18 South

Mayo Clinic

200 First St. SW

Rochester

MN 55905

USA

E-mail: ryu.jay@mayo.edu

Received:

Feb 092011

Accepted after revision:

May 142011

First published online:

June 302011 
In the thorax, ISD can manifest several patterns of involvement, some of which may cause severe respiratory compromise. Herein, we summarise the relevant aspects of ISD for pulmonologists, focusing on the patterns of intrathoracic involvement described to date and the favourable response of this disorder to corticosteroid therapy. We also discuss the evolving approach to the diagnosis and management of pulmonary ISD.

\section{GENERAL ASPECTS OF ISD}

ISD has been described mainly in adults, more often in males (70-80\%) than females, with a median age of 60-65 yrs (range 17-80 yrs) [1, 2, 21]. With the possible exception of those patients with predominantly head and neck involvement, in whom the male to female ratio is nearly equal [2], the epidemiological features appear to be similar regardless of the predominant organ involved.

Patients with ISD may present with a variety of symptoms referable to the organs involved, or with only abnormal imaging or laboratory findings (e.g. abnormal liver enzyme levels) in the absence of any symptoms [1-3]. Constitutional symptoms, such as fever and weight loss, can occur but are uncommon [1, 2].

Most patients (70-90\%) with ISD have an elevated serum IgG4 level $\left(>140 \mathrm{mg} \cdot \mathrm{dL}^{-1}\right)$, which is also found in $5 \%$ of the normal population [2, 20, 22]. Quantitatively, IgG4 is the smallest subclass of $\operatorname{IgG}$ and accounts for only $3-6 \%$ of total $\operatorname{IgG}$ in normal serum [22]. The IgG subclasses exhibit differences in their effector functions. Involvement of immunological mechanisms, possibly autoimmune in nature, seems likely in ISD, but exactly what role IgG4 plays in the pathogenesis of this disorder remains unclear $[2,22,23]$.

Our insights into the pathogenesis of ISD remain limited, partly because most studies to date on this issue relate mainly to autoimmune pancreatitis and Asian populations. Some genetic susceptibility factors, including human leukocyte antigen (HLA) haplotypes, as well as non-HLA genes (e.g. tumour necrosis factor- $\alpha$ promoter region haplotype), have been identified, although the findings have been inconsistent [24-29]. T-helper 2 cytokines, regulatory T-cells, interleukin-10 and transforming growth factor- $\beta$ are thought to play important roles in IgG4positive plasma cell infiltration and fibrogenesis [22, 23]. Although there are similarities in clinical and histological manifestations in patients with various forms of ISD, it is possible that there are some differences in the causation of lesions arising in different organs. It also remains unclear whether IgG4 truly mediates the disease in ISD, represents a protective response or is a bystander to the inflammatory process $[1,22]$.

Histopathological findings of ISD are characteristic, although not entirely specific, and may vary to some degree depending on the organ or site involved $[16,19]$. The shared features include lymphoplasmacytic inflammation, fibrosis, phlebitis and increased numbers of IgG4-positive plasma cells. Although these combined features are characteristic of ISD, no single histological feature is specific for the diagnosis of this disease $[16,19]$. The histopathological features of pulmonary ISD and the role of lung biopsy in the diagnostic evaluation will be discussed later in this article.

\section{INTRATHORACIC MANIFESTATIONS OF ISD}

In 2004, two separate case reports were published describing two males with parenchymal lung infiltrates and elevated serum IgG4 levels [30, 31]. One of these patients was a 63-yr-old male with autoimmune pancreatitis whose computed tomography (CT) scan of the chest revealed ground-glass opacities and honeycombing, predominantly in the lower lobes, and transbronchial lung biopsy demonstrated interstitial infiltration by lymphocytes and IgG4-positive plasma cells [30]. The second patient was a 38-yr-old male with autoimmune pancreatitis, retroperitoneal fibrosis and multiple nodular opacities in the lung [31]. His serum IgG4 level was also elevated and a surgical lung biopsy showed histopathological features of organising pneumonia. Both patients were treated with oral prednisone and, subsequently, had marked improvement of lung infiltrates.

Subsequent reports of pulmonary involvement in ISD (also referred to herein as intrathoracic ISD) have documented a broader spectrum of intrathoracic findings. The intrathoracic manifestations of ISD appear to be rather heterogeneous, resulting from involvement not only of the lung parenchyma, but also the intrathoracic lymph nodes, mediastinum and pleura, as well as the airways. In the context of these accumulating data, it seems plausible that ISD may account for a significant portion of several fibroinflammatory disorders of unknown cause encountered in pulmonary medicine, such as inflammatory pseudotumour of the lung, idiopathic interstitial pneumonias such as cryptogenic organising pneumonia, and fibrosing mediastinitis.

It is not known precisely how often pulmonary involvement occurs in patients with ISD. In a cross-sectional study of 114 patients with ISD, $16(14 \%)$ were found to have lung or pleural lesions [2]. In a separate retrospective study that reviewed imaging studies of 90 patients with autoimmune pancreatitis, $54 \%$ of patients were noted to have lung lesions [10]. Intrathoracic lymphadenopathy appears to be more common and can be detected in one-half or more of patients with ISD [10, 11, 15]. Intrathoracic manifestations of ISD have been reported in the presence or absence of one or more extrapulmonary lesions, such as autoimmune pancreatitis [2, 14, 16, 17, 32].

\section{CLINICAL FEATURES}

Respiratory symptoms including cough, exertional dyspnoea and chest pain have been described in approximately one-half of patients with pulmonary ISD, while the remaining patients presented with abnormal intrathoracic findings on imaging studies in the absence of respiratory symptoms [14, 17]. Constitutional symptoms, such as fever and weight loss, have been uncommon [14, 17]. Overall, the clinical presentation associated with pulmonary ISD appears to be rather nonspecific.

There are no studies assessing risk factors for pulmonary involvement in ISD. For example, it not known whether tobacco smoking or other inhalational exposures may increase the risk of pulmonary involvement in this systemic disorder.

\section{PATTERNS OF INTRATHORACIC INVOLVEMENT}

Intrathoracic involvement in ISD has seen described in the lung parenchyma, airways and pleura, as well as the mediastinum. Based on currently available data, intrathoracic manifestations can be categorised as shown in table 1 . Single or multiple manifestations can be identified in a single patient. For example, a lung nodule may be seen in combination with interstitial lung infiltrates and mediastinal lymphadenopathy. 


TABLE 1 Patterns of intrathoracic involvement in
immunoglobulin G4-related sclerosing disease
Parenchymal
Nodules or masses
Interstitial lung disease
Airways
Tracheobronchial stenosis
Pleural
Pleural nodules
Pleural effusion
Mediastinal
Lymphadenopathy
Fibrosing mediastinitis

\section{Parenchymal disease}

Lung parenchymal involvement in ISD consists mainly of rounded opacities (nodules $(\leqslant 3 \mathrm{~cm}$ in diameter) or masses $(>3 \mathrm{~cm}$ in diameter) $)$ and interstitial lung disease (ILD) [10, 14, 15, 17, 31, 33-37]. Rounded opacities (fig. 1) may appear solid or of ground-glass attenuation and range in size from $<1 \mathrm{~cm}$ to $>5 \mathrm{~cm}$ in diameter [10, 14, 15, 17, 33, 38]. Single or multiple rounded opacities may be demonstrated on chest radiography or CT with no particular predilection in terms of location $[14,15,17$, 33]. Rounded opacities commonly raise suspicion of malignancy, particularly when associated with spiculated margins [15, 17, 33]. Nodules of ground-glass attenuation may resemble bronchioalveolar carcinoma [17]. Thus, some patients with these types of lung lesions have undergone wedge resection or lobectomy for suspected malignancy.

Radiological features associated with ILD presentation have been varied on high-resolution CT of the chest (fig. 2). Earlier reports described bilateral interstitial lung infiltrates consisting of ground-glass attenuation in the mid- and lower lung zones, associated with honeycombing [30]. Subsequent reports have described a widening array of radiological patterns including patchy ground-glass opacities or consolidation, reticular opacities (irregular lines), honeycombing, and thickening of the bronchovascular bundles and interlobular septa [10, 14, 15, 17, 31, 33-37]. Pulmonary function testing reveals a reduced diffusing capacity and restrictive impairment, particularly in the presence of extensive parenchymal infiltrates [35, 39]. These features may resemble those found in idiopathic interstitial pneumonias, such as idiopathic pulmonary fibrosis (usual interstitial pneumonia), idiopathic nonspecific interstitial pneumonia, cryptogenic organising pneumonia and sarcoidosis [15, 17, 30, 31, 33].

\section{Airway disease}

There have been rare reports of airway disease associated with ISD. ITO et al. [40] described a 63-yr-old female with autoimmune pancreatitis who presented with cough and was noted to have tracheobronchial stenosis on bronchoscopic examination. The bronchial mucosa was noted to be oedematous and hypervascular, resembling the appearance seen in sarcoidosis. An obstructive pattern was seen on pulmonary function testing. CT scanning in this patient revealed intrathoracic lymphadenopathy and thickening of the bronchovascular bundle, also resembling the features seen in sarcoidosis.

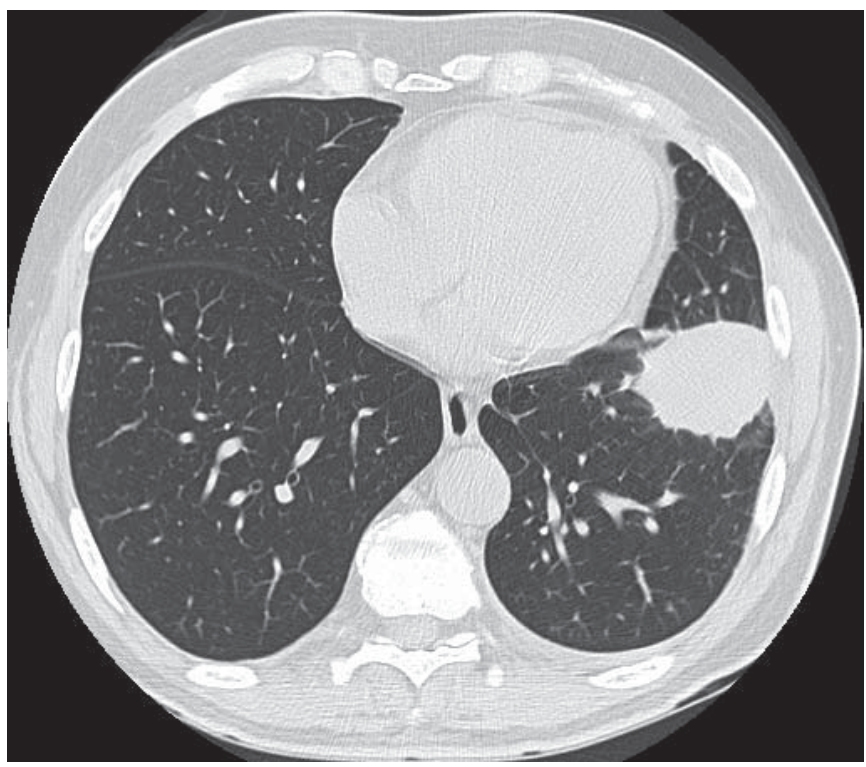

FIGURE 1. Computed tomography image of the chest of a 60 -yr-old male revealing a 5-cm left lung mass. Surgical lung biopsy showed an inflammatory pseudotumour with an increased number of immunoglobulin G4-positive cells. He had transient submandibular lymphadenopathy several months before but no other manifestations.

Another airway manifestation described with ISD has been extrinsic compression of the central airways due to fibrosing mediastinitis and bronchiectasis [14, 17, 41]. Bronchiectasis seen in this context appears to be that associated with parenchymal fibrosis in the peripheral zones of the lung, i.e. traction bronchiectasis, rather than involvement of the proximal large airways [14, 17].

\section{Pleural disease}

ZEN et al. [14] reported 21 patients with intrathoracic manifestations of ISD, of whom five (24\%) had predominantly pleural

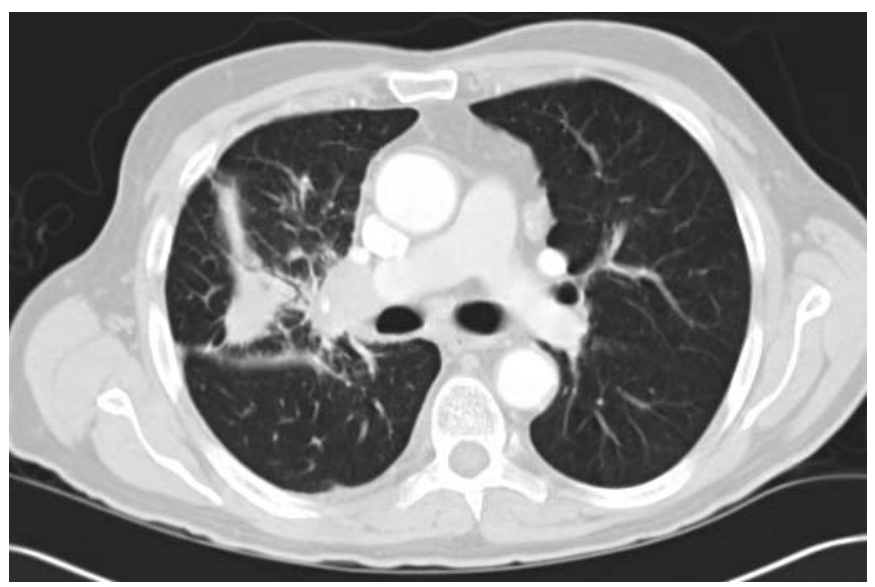

FIGURE 2. Computed tomography image of the chest of a 74-yr-old male demonstrating patchy consolidative infiltrate in the right lung. Surgical lung biopsy showed lymphoplasmacytic infiltrate with an increased number of immunoglobulin G4-positive cells. He had undergone a radical pancreaticoduodenectomy the year before for a pancreatic mass that proved to be autoimmune pancreatitis. 
disease. Pleural disease in these patients consisted of nodular lesions in the visceral or parietal pleura. Pleural effusion appears to be an uncommon feature in patients with pulmonary ISD but has been described as the main presentation in one patient [33]. However, pleuritis with fibrinous exudates and reactive changes have been relatively common histological findings on surgical lung biopsy in patients with intrathoracic ISD [16].

\section{Mediastinal disease}

Most common intrathoracic manifestation in ISD may be mediastinal and/or hilar lymphadenopathy, which have been described in $40 \%$ to $90 \%$ of patients with ISD, respectively (fig. 3) [9-11, 13-15]. In a survey of 65 patients with autoimmune pancreatitis, HAMANO et al. [11] reported hilar lymphadenopathy detected by CT scanning and gallium- 67 scintigraphy to be the most frequent extrapancreatic lesion (80\%). Other studies have identified intrathoracic lymphadenopathy in the majority of patients who undergo ${ }^{18} \mathrm{~F}$-fluoro-2-deoxy-D-glucose positron emission tomography $[42,43]$.

An unusual mediastinal manifestation of ISD is fibrosing mediastinitis. One case of fibrosing mediastinitis has been reported [41]. This patient improved with corticosteroid therapy, which is noteworthy, as fibrosing mediastinitis is generally considered to be a condition refractory to pharmacological therapy. It remains to be clarified what portion of fibrosing mediastinitis may represent ISD.

\section{HISTOPATHOLOGY}

Histopathological features associated with intrathoracic ISD are similar to those seen in extrapancreatic lesions with some exceptions. Features shared with extrapulmonary IgG4-related lesions include lymphoplasmacytic inflammation, fibrosis, phlebitis and increased numbers of IgG4-positive plasma cells (fig. 4) $[2,16,17,19,33]$. Plasma cells comprise the main cell type in the inflammatory infiltrates, followed by lymphocytes and histiocytes. Eosinophilic infiltration can be prominent but granulomas are rarely present and are usually small and vague

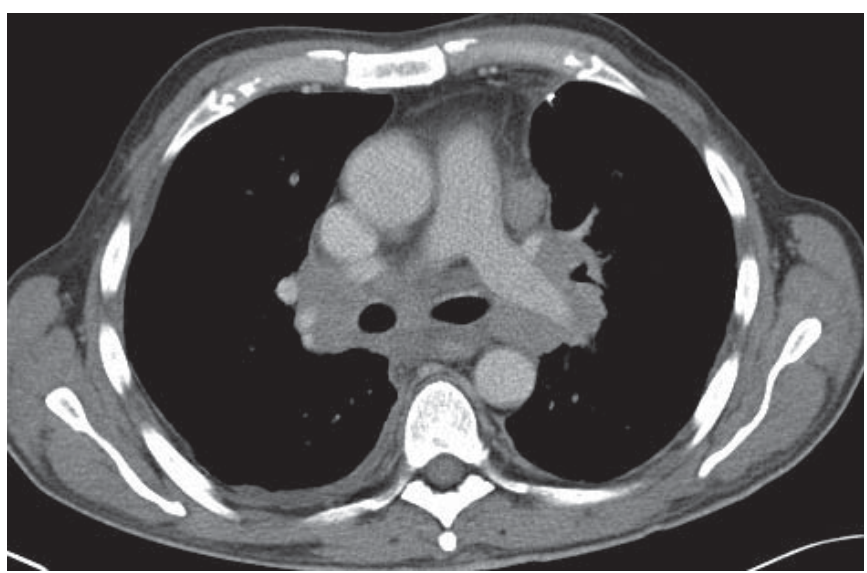

FIGURE 3. Computed tomography image of the chest of a 59-yr-old male showing mediastinal and bilateral hilar lymphadenopathy associated with mediastinal soft tissue thickening and a small right pleural effusion. Lymph node biopsy obtained by mediastinoscopy revealed findings consistent with immunoglobulin G4-related lymphadenopathy.
$[2,16]$. These changes are better appreciated on surgical lung biopsies but can also be identified on bronchoscopic and needle biopsies $[15,16]$. On surgical lung biopsies, prominent
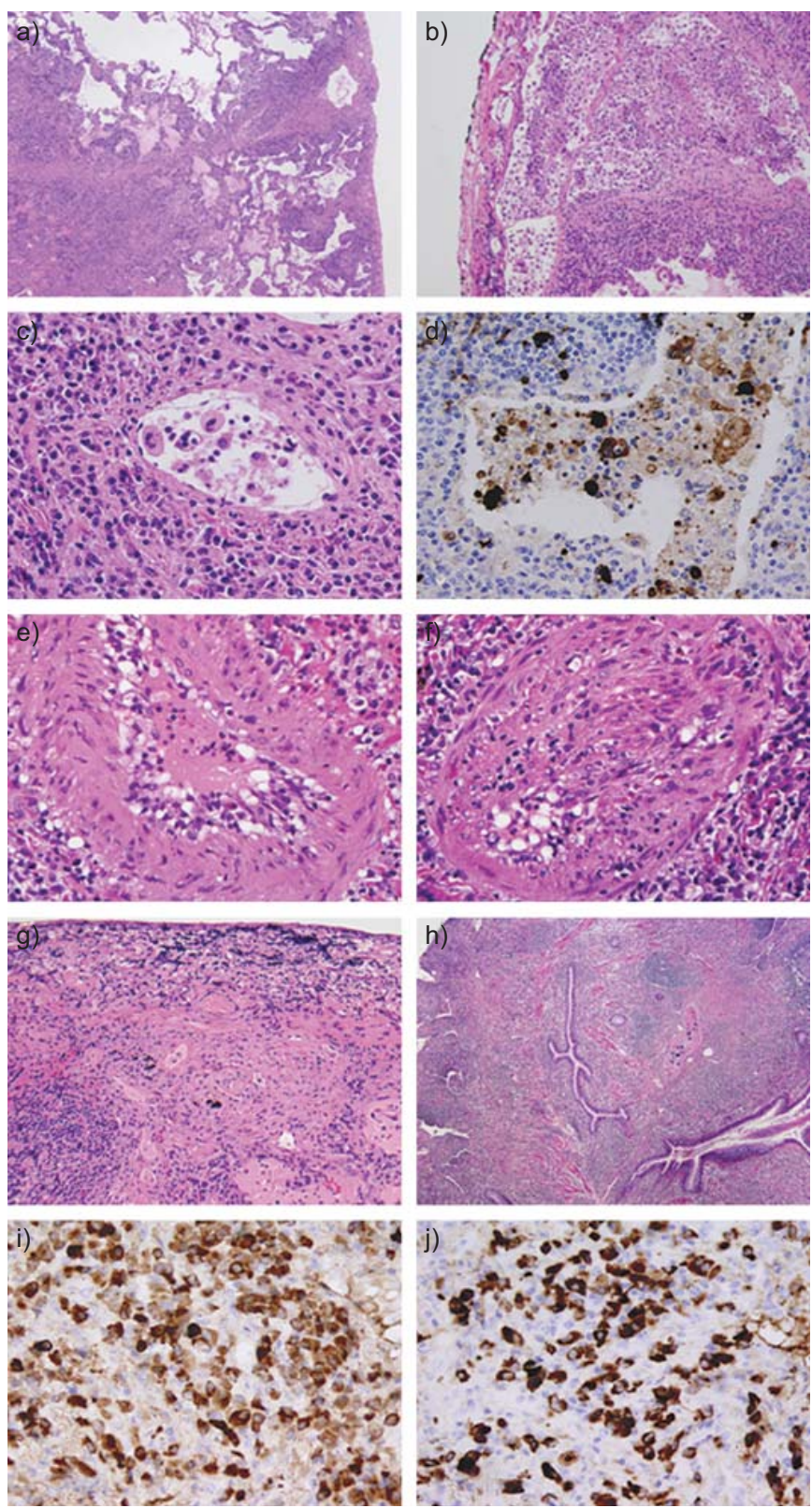

FIGURE 4. Pulmonary pathology in immunoglobulin (Ig)G4-related sclerosing disease. a) Lower power view of the lung biopsy showing a lymphangitic distribution of fibroinflammatory changes with focal consolidation shown in the lower field (haematoxylin and eosin (HE), $40 \times$ original magnification). b) Dilated lymphatic spaces filled with histiocytes seen in the visceral pleura, which shows mild fibrinous pleuritis (HE, $100 \times$ ). c) and d) Emperipolesis in S100-positive histiocytes (HE and S100, $400 \times)$. e) Endothelialitis characterised by subendothelial mixed inflammatory infiltrates with reactive endothelial cell changes (HE, $400 \times)$. f) Intimal and mural inflammatory infiltrates accompanied by luminal occlusion owing to cellular myointimal proliferation $(\mathrm{HE}, 400 \times)$. g) Fibroblastic proliferation in the centre surrounded by mononuclear infiltrates at the periphery of field (HE, 100 $\times$ ). h) Peribronchial inflammation without structural alteration of the involved airways $(H E, 40 \times)$. i) and j) A marked increase in IgG and IgG4-positive plasma cells among the inflammatory infiltrates showing a high $\lg G 4 / \operatorname{lgG}$ ratio (IgG and IgG4, 400 $\times$ ). Reproduced from [16] with permission from the publisher. 
lymphangitic distribution involving the interlobular septa and visceral pleura is seen [16]. Dilated lymphatic spaces contain histiocytes showing emperipolesis of lymphocytes [16].

In contrast to histopathological findings seen in the pancreas in autoimmune pancreatitis, characteristic storiform fibrosis is not as apparent in the lung where collagenised fibrosis and active fibroblastic proliferation are more prominent [16]. In addition, both pulmonary arteries and veins are involved by intimal and mural inflammation in contrast to the findings in the involved pancreas, which show obliterative phlebitis with sparing of the arteries [16]. Necrotising vasculitis is not seen in ISD.

An increased number of IgG4-positive plasma cells is seen in regional and nonregional lymph nodes in patients with ISD. However, other histopathological features may be relatively nonspecific and have been broadly divided into three patterns: 1) Castleman disease-like; 2) follicular hyperplasia; and 3) interfollicular expansion [13]. In the lung parenchyma, histopathological features of organising pneumonia and nonspecific interstitial pneumonia have been described in patients with IgG4-related pulmonary disease [16, 31, 35, 39].

\section{LABORATORY TESTS}

Serum IgG4 level is elevated $\left(>140 \mathrm{mg} \cdot \mathrm{dL}^{-1}\right)$ in the majority of patients with intrathoracic ISD [14, 16, 17]. The sensitivity and specificity of serum IgG4 level with respect to intrathoracic ISD are not precisely known but appear to be similar to those associated with ISD in general, as described earlier in this article.

There are no other blood tests that are applicable to pulmonary involvement in ISD other than perhaps serum Kerbs von den Lungen-6 (KL-6) level. KL-6 is a circulating high-molecular weight glycoprotein (MUC1 mucin) expressed by type II pneumocytes in the lung. Serum KL-6 level has been reported to be elevated in patients with various parenchymal lung diseases such as idiopathic pulmonary fibrosis [44-46]. Circulating KL-6 level has been suggested to correlate with the extent of lung injury and have prognostic value but is not widely available outside of Japan. HIRANO et al. [35] identified pulmonary involvement in four out of 30 patients with autoimmune pancreatitis and the circulating level of KL-6 was elevated in all four patients. The utility of serum KL-6 level in detecting pulmonary involvement in patients with ISD remains to be determined.

Analysis of bronchoalveolar lavage (BAL) fluid obtained by bronchoscopy has been reported to show increased levels of IgG4 when compared with the specimens obtained from patients with sarcoidosis [15]. The BAL IgG4 level was observed to correlate with the serum IgG4 level. BAL cellular analysis typically reveals lymphocytosis as expected based on histopathological findings $[15,40]$. Overall, the BAL data relating to IgG4-related pulmonary disease are sparse and the role of BAL in the evaluation of patients with ISD remains to be clarified.

\section{DIAGNOSIS OF INTRATHORACIC ISD}

It appears that intrathoracic manifestations of ISD can be encountered in the presence or absence of extrathoracic lesions, such as autoimmune pancreatitis. In patients who already have an established diagnosis of ISD, concern regarding pulmonary involvement may arise when intrathoracic abnormalities are detected in such patients. As intrathoracic manifestations of
ISD are so varied, virtually any type of intrathoracic finding may need to be evaluated for this possibility. Histopathological examination of tissue biopsy from the intrathoracic lesion will be useful in distinguishing manifestations related to ISD from a separate process, such as lung cancer, lymphoma or sarcoidosis. The method of biopsy will depend on the type, location and distribution of the target lesion as well as the clinical context, e.g. comorbid factors.

Although the presence of IgG4-positive lymphoplasmacytic infiltrate is characteristic of ISD, it is not entirely specific for the diagnosis of ISD either in the thorax or elsewhere [16, 19]. In addition, there is no single histopathological parameter that distinguishes IgG4-related pulmonary disease from other similarly appearing pathological processes. Based on our experience to date, we propose that the histopathological lesion of intrathoracic ISD is characterised by a fibroinflammatory process (in the absence of an infection), with or without nodule formation, exhibiting plasma cells that comprise $>50 \%$ of inflammatory infiltrates with endothelialitis in the pulmonary arteries and/or veins [16]. IgG4-positive cells are increased and account for $>30 \%$ of IgG-positive cells on immunohistochemical staining $[14,16,17]$. Interlobular septal and pleural involvement is commonly present. As these histopathological findings may not be specific for ISD, it is crucial that these findings be correlated with the clinical and imaging context (e.g. serum IgG4 level and presence of extrapulmonary lesions suggestive of ISD). In the presence of typical histopathological findings and compatible clinico-radiological features that do not suggest another disorder, a diagnosis of ISD can be made. Additional studies are needed to identify features that distinguish ISD from other disorders that may share some of the described characteristics and to identify standard diagnostic criteria.

Intrathoracic ISD can be seen before, simultaneously or after the diagnosis of autoimmune pancreatitis or other extrathoracic lesions of ISD. In patients without a known diagnosis of ISD, possibility of intrathoracic ISD could be overlooked as the clinical and radiological manifestations of this disorder are relatively nonspecific. Histopathological examination including IgG4 immunostaining of biopsy specimens and correlation with clinical, laboratory and imaging findings would be crucial in arriving at the correct diagnosis.

Although serum IgG4 level will be elevated in the majority of patients with ISD, including those with intrathoracic disease, the absence of this finding does not exclude this diagnosis. BAL fluid analysis has been reported to show elevated IgG4 levels and lymphocytosis, but the diagnostic utility of these measures remains to be defined.

\section{TREATMENT}

As with extrathoracic lesions of ISD, intrathoracic lesions associated with ISD generally responds well to corticosteroid therapy $[11,13,14,16,17,30,31,33,35,36,38,40,42,43]$. This positive response to corticosteroid therapy is observed whether the predominant disease is in the lung itself, in the airway, the pleura or the mediastinum and emphasises the importance of diagnosing ISD. The exact regimen of corticosteroids is not specified in many of the studies to date but typically consists of oral prednisone begun at a dose of between $30 \mathrm{mg} \cdot$ day $^{-1}$ to $1 \mathrm{mg} \cdot \mathrm{kg}^{-1} \cdot \mathrm{day}^{-1}$. Favourable response is usually observed by 
2 weeks on treatment. After 1-2 weeks, the prednisone dose is gradually decreased over the following several months with continued monitoring for complete resolution and possible recurrence during the interval.

The optimal dose and duration of corticosteroid therapy in the treatment of ISD remains to be defined. A retrospective study on patients with autoimmune pancreatitis demonstrated a low maintenance dose of prednisone $\left(\leqslant 10 \mathrm{mg} \cdot\right.$ day $\left.^{-1}\right)$ to be associated with a significantly lower rate of relapse [21]. These results have been extrapolated by some authors to patients with extrapancreatic ISD. Thus, a low maintenance dose of prednisone has been used for some patients beyond several months of treatment to reduce the risk of relapse. Spontaneous improvement in intrathoracic ISD has not been described. Although corticosteroid therapy is used widely in patients with ISD, it is probably not needed in the management of patients who undergo complete surgical resection of focal lesions, such as a solitary pulmonary nodule or mass [14, 38].

There is relatively little data on the use of pharmacological agents other than corticosteroids in the treatment of ISD. The use of bortezomib, a proteasome inhibitor, and the addition of cyclosporine to corticosteroid therapy have been reported in two separate case reports describing patients with recurrent IgG4related pulmonary disease; this treatment appeared to provide benefit $[34,47]$. In extrathoracic ISD, azathioprine, mycophenolate, methotrexate and cyclophosphamide have sometimes been used to prevent long-term relapse [8, 48]. Recently, rituximab therapy was reported to result in a rapid decline of serum IgG4 levels and prompt clinical improvement in four patients with IgG4-related systemic disease [48]. In this study, rituximab was used as a corticosteroid-sparing agent.

\section{PROGNOSIS}

Although the response to corticosteroid therapy is favourable in most patients with intrathoracic ISD, long-term follow-up data are currently not available. These patients may develop extrathoracic lesions of ISD in the months and years following their diagnosis [2]. In addition, some patients with intrathoracic ISD may not experience complete resolution of their disease and have persistent radiological abnormalities. ZEN et al. [14] described residual radiological abnormalities in three of their 21 patients with IgG4-related pulmonary and pleural disease after treatment.

Association with malignancy has been described in patients with ISD. These malignancies have included lymphoma and pancreatic cancer, as well as lung cancer $[2,13,14,49,50]$. It remains to be clarified whether there truly is an increased risk of malignancy in patients with ISD.

\section{CONCLUSIONS}

Intrathoracic manifestations of ISD are varied and have been described in the lung parenchyma, airway and pleura, as well as the mediastinum. Although data to date suggest that ISD may account for a portion of various idiopathic fibroinflammatory conditions encountered in the chest, including inflammatory pseudotumours, idiopathic interstitial pneumonias, fibrosing mediastinitis, inflammatory pleural lesions and occasionally airway disease, some of these attributed manifestations remain controversial and additional studies are needed to clarify the relationship. The pathogenesis, diagnostic criteria and the relevance of ISD to pulmonary medicine need to be investigated further.

\section{STATEMENT OF INTEREST}

None declared.

\section{REFERENCES}

1 Khosroshahi A, Stone JH. A clinical overview of IgG4-related systemic disease. Curr Opin Rheumatol 2011; 23: 57-66.

2 Zen Y, Nakanuma YL. IgG4-related disease: a cross-sectional study of 114 cases. Am J Surg Pathol 2010; 34: 1812-1819.

3 Kamisawa T, Okamoto A. IgG4-related sclerosing disease. World J Gastroenterol 2008; 14: 3948-3955.

4 Neild GH, Rodriguez-Justo M, Wall C, et al. Hyper-IgG4 disease: report and characterisation of a new disease. BMC Med 2006; 4: 23.

5 Yoshida K, Toki F, Takeuchi T, et al. Chronic pancreatitis caused by an autoimmune abnormality. Proposal of the concept of autoimmune pancreatitis. Digest Dis Sci 1995; 40: 1561-1568.

6 Hamano H, Kawa S, Horiuchi A, et al. High serum IgG4 concentrations in patients with sclerosing pancreatitis. $N$ Engl J Med 2001; 344: 732-738.

7 Deshpande V, Gupta R, Sainani N, et al. Subclassification of autoimmune pancreatitis: a histologic classification with clinical significance. Am J Surg Pathol 2011; 35: 26-35.

8 Ghazale A, Chari ST, Zhang L, et al. Immunoglobulin G4associated cholangitis: clinical profile and response to therapy. Gastroenterol 2008; 134: 706-715.

9 Naitoh I, Nakazawa T, Ohara H, et al. Clinical significance of extrapancreatic lesions in autoimmune pancreatitis. Pancreas 2010; 39: e1-e5.

10 Fujinaga Y, Kadoya M, Kawa S, et al. Characteristic findings in images of extra-pancreatic lesions associated with autoimmune pancreatitis. Eur J Radiol 2010; 76: 228-238.

11 Hamano $\mathrm{H}$, Arakura $\mathrm{N}$, Muraki $\mathrm{T}$, et al. Prevalence and distribution of extrapancreatic lesions complicating autoimmune pancreatitis. J Gastroenterol 2006; 41: 1197-1205.

12 Stone JH, Khosroshahi A, Deshpande V, et al. IgG4-related systemic disease accounts for a significant proportion of thoracic lymphoplasmacytic aortitis cases. Arthritis Rare Res 2010; 62: 316-322.

13 Cheuk W, Yuen HKL, Chu SYY, et al. Lymphadenopathy of IgG4related sclerosing disease. Am J Surg Pathol 2008; 32: 671-681.

14 Zen Y, Inoue D, Kitao A, et al. IgG4-related lung and pleural disease: a clinicopathologic study of 21 cases. Am J Surg Pathol 2009; 33: 1886-1893.

15 Tsushima K, Tanabe T, Yamamoto H, et al. Pulmonary involvement of autoimmune pancreatitis. Eur J Clin Invest 2009; 39: 714-722.

16 Shrestha B, Sekiguchi H, Colby TV, et al. Distinctive pulmonary histopathology with increased IgG4-positive plasma cells in patients with autoimmune pancreatitis: report of 6 and 12 cases with similar histopathology. Am J Surg Pathol 2009; 33: 1450-1462.

17 Inoue $\mathrm{D}$, Zen $\mathrm{Y}, \mathrm{Abo} \mathrm{H}$, et al. Immunoglobulin G4-related lung disease: CT findings with pathologic correlations. Radiology 2009; 251: $260-270$.

18 Pickartz T, Mayerle J, Lerch MM. Autoimmune pancreatitis. Nat Clin Pract Gastroenterol Hepatol 2007; 4: 314-323.

19 Smyrk TC. Pathological features of IgG4-related sclerosing disease. Curr Opin Rheumatol 2011; 23: 74-79.

20 Sah RP, Chari ST. Serologic issues in IgG4-related systemic disease and autoimmune pancreatitis. Curr Opin Rheumatol 2011; 23: 108-113.

21 Kamisawa T, Shimosegawa T, Okazaki K, et al. Standard steroid treatment for autoimmune pancreatitis. Gut 2009; 58: 1504-1507.

22 Nirula A, Glaser SM, Kalled SL, et al. What is IgG4? A review of the biology of a unique immunoglobulin subtype. Curr Opin Rheumatol 2011; 23: 119-124. 
23 Zen Y, Nakanuma Y. Pathogenesis of IgG4-related disease. Curr Opin Rheumatol 2011; 23: 114-118.

24 Kawa S, Ota M, Yoshizawa K, et al. HLA DRB10405-DQB10401 haplotype is associated with autoimmune pancreatitis in the Japanese population. Gastroenterology 2002; 122: 1264-1269.

25 Park DH, Kim M-H, Oh HB, et al. Substitution of aspartic acid at position 57 of the DQbeta1 affects relapse of autoimmune pancreatitis. Gastroenterol 2008; 134: 440-446.

26 Scalapino KJ, Daikh DI. CTLA-4: a key regulatory point in the control of autoimmune disease. Immunol Rev 2008; 223: 143-155.

27 Chang $\mathrm{M}-\mathrm{C}$, Chang $\mathrm{Y}-\mathrm{T}$, Tien $\mathrm{Y}-\mathrm{W}$, et al. T-cell regulatory gene CTLA-4 polymorphism/haplotype association with autoimmune pancreatitis. Clin Chem 2007; 53: 1700-1705.

28 Umemura $\mathrm{T}$, Ota $\mathrm{M}$, Hamano $\mathrm{H}$, et al. Association of autoimmune pancreatitis with cytotoxic T-lymphocyte antigen 4 gene polymorphisms in Japanese patients. Am J Gastroenterol 2008; 103: 588-594.

29 Umemura T, Ota M, Hamano H, et al. Genetic association of Fc receptor-like 3 polymorphisms with autoimmune pancreatitis in Japanese patients. Gut 2006; 55: 1367-1368.

30 Taniguchi T, Ko M, Seko S, et al. Interstitial pneumonia associated with autoimmune pancreatitis. Gut 2004; 53: 770.

31 Duvic C, Desrame J, Lêvéque C, et al. Retroperitoneal fibrosis, sclerosing pancreatitis and bronchiolitis obliterans with organizing pneumonia. Nephrol Dial Transpl 2004; 19: 2397-2399.

32 Shigemitsu H, Koss MN. IgG4-related interstitial lung disease: a new and evolving concept. Curr Opin Pulm Med 2009; 15: 513-516.

33 Yamashita K, Haga H, Kobashi Y, et al. Lung involvement in IgG4related lymphoplasmacytic vasculitis and interstitial fibrosis: report of 3 cases and review of the literature. Am J Surg Pathol 2008; 32: 1620-1626.

34 Kobayashi H, Shimokawaji T, Kanoh S, et al. IgG4-positive pulmonary disease. J Thor Imag 2007; 22: 360-362.

35 Hirano K, Kawabe T, Komatsu Y, et al. High-rate pulmonary involvement in autoimmune pancreatitis. Intern Med J 2006; 36: 58-61.

36 Taniguchi T, Hamasaki A, Okamoto M. A case of suspected lymphocytic hypophysitis and organizing pneumonia during maintenance therapy for autoimmune pancreatitis associated with autoimmune thrombocytopenia. Endocr J 2006; 53: 563-566.

37 Ohara H, Nakazawa T, Sano $\mathrm{H}$, et al. Systemic extrapancreatic lesions associated with autoimmune pancreatitis. Pancreas 2005; 31: 232-237.
38 Zen Y, Kitagawa S, Minato H, et al. IgG4-positive plasma cells in inflammatory pseudotumor (plasma cell granuloma) of the lung. Human Pathol 2005; 36: 710-717.

39 Takato H, Yasui M, Ichikawa $Y$, et al. Nonspecific interstitial pneumonia with abundant IgG4-positive cells infiltration, which was thought as pulmonary involvement of IgG4-related autoimmune disease. Intern Med 2008; 47: 291-294.

40 Ito $\mathrm{M}$, Yasuo $\mathrm{M}$, Yamamoto $\mathrm{H}$, et al. Central airway stenosis in a patient with autoimmune pancreatitis. Eur Respir J 2009; 33: 680-683.

41 Inoue $\mathrm{M}$, Nose $\mathrm{N}$, Nishikawa $\mathrm{H}$, et al. Successful treatment of sclerosing mediastinitis with a high serum IgG4 level. Gen Thorac Cardiovas Surg 2007; 55: 431-433.

42 Nakajo M, Jinnouchi S, Fukukura Y, et al. The efficacy of wholebody FDG-PET or PET/CT for autoimmune pancreatitis and associated extrapancreatic autoimmune lesions. Eur J Nucl Med Mol Imag 2007; 34: 2088-2095.

43 Hamed G, Tsushima K, Yasuo M, et al. Inflammatory lesions of the lung, submandibular gland, bile duct and prostate in a patient with IgG4-associated multifocal systemic fibrosclerosis. Respirology 2007; 12: 455-457.

44 Yokoyama A, Kondo K, Nakajima M, et al. Prognostic value of circulating KL-6 in idiopathic pulmonary fibrosis. Respirology 2006; 11: 164-168.

45 Al-Salmi QA, Walter JN, Colasurdo GN, et al. Serum KL-6 and surfactant proteins $\mathrm{A}$ and $\mathrm{D}$ in paediatric interstitial lung disease. Chest 2005; 127: 403-407.

46 Kinoshita F, Hamano H, Harada H, et al. Role of KL-6 in evaluating the disease severity of rheumatoid lung disease: comparison with HRCT. Respir Med 2004; 98: 1131-1137.

47 Khan ML, Colby TV, Viggiano RW, et al. Treatment with bortezomib of a patient having hyper IgG4 disease. Clin Lymphoma Myeloma Leuk 2010; 10: 217-219.

48 Khosroshahi A, Bloch DB, Deshpande V, et al. Rituximab therapy leads to rapid decline of serum IgG4 levels and prompt clinical improvement in IgG4-related systemic disease. Arthritis Rheum 2010; 62: 1755-1762.

49 Takahashi N, Ghazale AH, Smyrk TC, et al. Possible association between IgG4-associated systemic disease with or without autoimmune pancreatitis and non-Hodgkin lymphoma. Pancreas 2009; 38: $523-526$

50 Fukui T, Mitsuyama T, Takaoka M, et al. Pancreatic cancer associated with autoimmune pancreatitis in remission. Intern Med 2008; 47: 151-155. 